\title{
Chronic kidney disease is a risk factor for cardiovascular disease
}

\section{Author: XX Swanepoe}

\author{
Address for correspondence: \\ Dr XX Swanepoel \\ $X x$ \\ $x \times$ \\ $x \times$ \\ 0000 \\ Email: \\ xxxxxxxxxxx@sun.ac.za
}

ABSTRACT Chronic kidney disease (CKD) is common, harmful and treatable.There are no accurate statistics on the prevalence of kidney disease in South Africa but the world prevalence is estimated at approximately $10 \%$. Patients with CKD have cardiovascular (CV) mortality rates as high as $\mathbf{5 0 \%}$. In addition to traditional risk factors, these patients are exposed to other non-traditional CV risk factors. It is important to stress that with the development of early CKD in the form of increasing albuminuria, even from within the normal range (a marker of endothelial dysfunction), the patient is at increased risk of coronary heart disease.

"Reverse epidemiology" is the paradox where a high body mass index predicts a better long-term survival in patients with CKD on haemodialysis. Curiously these patients have increased inflammatory markers and atherosclerosis. "Confounding by disease" or "reverse causality" is used to explain why the traditional relationship of increased cholesterol, increased LDL cholesterol and CV risk is not present (except in nephrotics) in CKD.

The high phosphate level predisposes to calcium phosphate crystal deposition in the vessels and heart valves. The resultant arteriosclerosis produces the increased pulse wave velocity and systolic hypertension. Rapid valvular calcification, aortic stenosis being a particular difficulty, and early onset coronary artery calcification occur.

Arrhythmias are common and the long QT interval syndrome is particularly pertinent. The risk of acquired LQTS is increased by the administration of drugs. Cardiac troponin T is elevated in approximately $25 \%$ of asymptomatic patients with CKD.

The use of angiotensin converting enzyme inhibitors and angiotensin receptor blockers have been shown to reduce proteinuria and to slow the progressive loss of renal function seen in CKD. These agents also improve cardiac outcomes and reduce the incidence of stroke.

Most trials on the management of coronary artery disease have excluded patients with renal disease, and it remains unclear whether the results obtained from these trails can be extrapolated to patients with CKD.

\section{INTRODUCTION}

Chronic kidney disease (CKD) is a condition in which a pathological process affects components of the kidney for longer than three months When the diagnosis of CKD is made, glomerular filtration rate (GFR) may still be normal.The KJDOQI guidelines ${ }^{(1)}$ of the US National Kidney Foundation have put forward a staging of CKD where stage I comprises a normal glomerular filtration rate $(>90 \mathrm{ml} / \mathrm{min})$ and stage 5 or ESRD describes the patient with a GFR of $<15 \mathrm{ml} / \mathrm{min}$ (Table I).

\begin{tabular}{|c|c|c|}
\hline Stage & Description & GFR $\left(\mathrm{ml} / \mathrm{min} / 1.73 \mathrm{~m}^{2}\right)$ \\
\hline । & Kidney damage + normal or $\uparrow$ GFR & $\geq 90$ \\
\hline 2 & Mild $\downarrow$ in GFR & $60-89$ \\
\hline 3 & Moderate $\downarrow$ in GFR & $30-59$ \\
\hline 4 & Severe $\downarrow$ in GFR & $15-29$ \\
\hline 5 & Kidney failure & $<15$ \\
\hline
\end{tabular}

* Adapted from Kidney Disease Outcomes Quality Initiative, Am J Kidney Dis Suppl I 2002;39:SI-S246.

There are no accurate statistics on the prevalence of kidney disease in South Africa, but the literature suggests a prevalence of around $10 \%$ in the adult population throughout the world. ${ }^{(2)}$

\section{CARDIOVASCULAR DISEASE IN PATIENTSWITH CKD}

The Framingham data on cardiovascular disease (CV) risk factors, i.e. hypertension, smoking, diabetes, dyslipidaemia and left ventricular hypertrophy applies to the majority of the population with CKD. In addition to these traditional factors, the patient with CKD is exposed to other cardiovascular risk factors, e.g. anaemia, bone-mineral disorder, inflammation and oxidative stress.(3) The prevalence of these "nontraditional" risk factors increases as GFR worsens (Table 2).

TABLE 2: Risk factors for cardiovascular disease (CVD) in chronic kidney disease (CKD)

\section{Classical}

Diabetes

Smoking

Dyslipidaemia

Sedentary Lifestyle

Obesity
Non classical - related to kidney disease

\begin{tabular}{ll} 
Hypervolaemia & Homocysteine \\
\hline Anaemia & Inflammation \\
\hline Bone mineral disorder & Oxidative Stress \\
\hline $\begin{array}{l}\text { Hyperdynamic circulation } \\
\text { due to large A-V fistulae }\end{array}$ & \\
\hline
\end{tabular}


As a result, patients with CKD are commonly affected by excess CV morbidity and mortality. ${ }^{(4,5)} \mathrm{CV}$ mortality rates may be as high as $50 \%$. Even stable patients with a moderate degree of severity (CKD stage 3 , GFR $<60 \mathrm{ml} / \mathrm{min}$ ) have a high prevalence of CVD. ${ }^{(6)}$ Goicoechea et al. showed that age and previous coronary artery disease, the traditional $\mathrm{CV}$ risk factors, accurately predicted cardiovascular events in these patients ${ }^{(6)}$.

Although the subsequent discussion will focus on those patients with a GFR $<60 \mathrm{ml} / \mathrm{min}$, it is important to stress that, even at an early stage, with increasing amounts of albuminuria (from within the low normal range) to the subsequent development of overt proteinuria, the patient is at increasing risk of coronary heart disease. ${ }^{(7.8)}$ It appears that this albuminuria/proteinuria is independent of traditional risk factors and is a marker of endothelial dysfunction. It is postulated that it is this endothelial cell dysfunction that permits entry of atherogenic lipoproteins into the media of the blood vessels. ${ }^{(9)}$

\section{TRADITIONAL CARDIOVASCULAR RISK FACTORS}

\section{Obesity}

There is a paradox, called "reverse epidemiology", wherein a high body mass index (BMI) is better for long-term survival in patients on chronic haemodialysis. ${ }^{(10)}$ Beddhu has shown that haemodialysis patients with a high BMI have increased inflammatory markers (elevated CRP levels) and atherosclerosis. ${ }^{(10)}$ Yet their survival is better than those with a lower BMI. The phenomenon is explained by hypothesising that good nutrition overwhelms the negative impact of atherosclerosis. Malnutrition, on the other hand, offers no "buffer" for these patients and is a risk factor for cardiovascular disease. Others suggest that this "risk factor paradox" could be as a result of absorption into the adipose tissue of uraemic toxins or as a result of a more stable haemodynamic state, or unique neurohormonal constellations or reverse causation. ${ }^{(11)}$.

\section{Dyslipidaemia}

There are numerous publications on the pathogenesis of the dyslipidaemia of CKD. A summary of proposed causative mechanisms includes the existence of insulin resistance, the presence of low oncotic pressure stimulation of the hepatocytes, as well as delayed metabolism because of reduced lipoprotein lipase and hepatic triglyceride lipase activity. The pool of lipoprotein lipase is reduced in nephrotic syndrome patients. Malnutrition plays a role in reducing the cholesterol level in CKD patients.

The important disturbances in serum lipids and apoproteins are shown in Table 3. From this table it can clearly be seen that increased cholesterol, increased LDL cholesterol and low HDL is not found in
TABLE 3: Dyslipidaemia in chronic kidney disease (CKD)

\begin{tabular}{ll}
\multicolumn{1}{c|}{ Nephrotic } & \multicolumn{1}{c}{ Non-nephrotic } \\
\hline$\uparrow$ Cholesterol & Normal Cholesterol \\
$\uparrow$ Triglycerides & $\uparrow$ Triglycerides \\
\hline$\uparrow$ LDLCholesterol & Normal/mildly $\uparrow$ LDL Cholesterol \\
\hline $\begin{array}{l}\text { apo B, C-II and E } \\
\uparrow\end{array}$ & $\uparrow$ apo (a) \\
\hline $\boldsymbol{H}$ HDL Cholesterol & $\downarrow$ or normal HDL Cholesterol \\
\hline
\end{tabular}

CKD patients (except in nephrotics). However, despite normal or low levels of LDL cholesterol and total cholesterol, atherosclerosis is very prevalent in these patients. Baigent and Landray ascribe this phenomenon to "confounding by disease" or "reverse causality".(12) In support of "confounding by disease" they point to work done by Sarnak and Levey who show that the association of many risk factors, in concert, impinge on cardiovascular mortality/morbidity. ${ }^{(13)}$ The point is made that, in this soup of metabolic derangement, the LDL cholesterol need not be abnormally high to be a CV risk factor.

There is increasing evidence to show that lipoprotein(a) is degraded by the normal functioning kidney. Consequently levels increase with deteriorating renal function. Elevated levels of lipoprotein(a) are associated with an increased incidence of atherosclerotic events in dialysis patients. ${ }^{(14)}$

\section{Hypertension}

This must be the most important traditional risk factor associated with CVD. Not only is the incidence of stroke and CVD increased in the presence of hypertension ${ }^{(15)}$, but CKD progression to end-stage is accelerated by uncontrolled blood pressure.(I)

Lewington et al. demonstrated a doubling of risk for stroke and coronary artery disease for every $20 \mathrm{~mm} \mathrm{Hg}$ increase in systolic pressure above $120 \mathrm{mmHg}$. 15$)$

The diseased kidney has an increased propensity to retain sodium. It has been suggested that the kidney is the universal cause of hypertension. For example, merely having a reduced number of nephrons predisposes that individual to the development of hypertension. ${ }^{(16,17)}$ This situation is found in those with low birth weights.

\section{NON-TRADITIONAL CARDIOVASCULAR RISK FACTORS}

\section{Bone-mineral disorder (BMD)}

The diseased kidney's inability to excrete excessive dietary inorganic phosphate is the major cause of the BMD. In addition, the accompanying 
inability of the diseased kidney to hydroxylate 25-hydroxycholecalcifero to active Vitamin D3, limits calcium absorption with consequent hypocalcaemia. These three abnormalities, i.e. hyperphosphataemia, hypocalcaemia and decreased levels of active Vitamin D3, will result in the increased release of parathyroidhormone (PTH). PTH mobilizes calcium and phosphate from the gut and bone which will aggravate an already hyperphosphataemic state.

The increase in phosphate levels predisposes to the precipitation of calcium phosphate crystals in the media of the walls of the vessels. ${ }^{(18,19)}$ In an elegant study on the rat model,Verberckmoes et al. found calcium phosphate and whitlockite crystals in the aortas of uraemic animals. ${ }^{(18)}$ The use of $X$-ray fluorescence mapping with integration of the diffraction patterns in the scanned areas in the rats' aortas, revealed calcium phosphate $\left(\mathrm{CaHPO}_{4}, 2 \mathrm{H}_{2} \mathrm{O}\right)$ as an amorphous substance, apatite $\mathrm{Ca}_{10}\left(\mathrm{PO}_{4}\right)_{6}(\mathrm{OH})_{2}$ and whitlockite $\left[(\mathrm{Mg}, \mathrm{Ca})_{3}(\mathrm{PO} 4)\right]_{2}$. Whitlockite and apatite are insoluble. ${ }^{(19)}$ Whitlockite was found only in those animals treated with Vitamin D3. Vitamin D3 increases the absorption of magnesium $(\mathrm{Mg}$ ) which subsequently then favours the formation of the insoluble whitlockite.

High phosphate levels also stimulate the differentiation of vascular smooth muscle cells into osteoblasts. ${ }^{(20)}$ Therefore, the CKD patient has the ability to form "bone" in the vessels. Increased Ca/Phosphate product, hyperparathyroidism and increased phosphate levels have been associated with an increased risk of coronary artery calcification. (21) The data published by Goodman et al. confirmed the prevalence of early coronary artery calcification, as determined by electron beam tomography, in young adults on dialysis. They also pointed to the dangers of calcium carbonate as a phosphate binder, a high calcium/phosphate product and length of time on dialysis.

The development of medial and intimal sclerosis in vessel walls results in both arteriosclerosis and atherosclerosis. The resultant "stiff" wall is non-compliant, producing an increased pulse wave velocity and systolic hypertension. It is not unusual to see CKD patients treated on dialysis with blood pressure readings of $210 / 70 \mathrm{mmHg}$.

Figure I illustrates an example of both large and small vessel calcification. A rare complication of small vessel calcification produces systemic calciphylaxis, with skin infarction and progressive ischemic destruction of the subcutaneous tissue (Figure 2). Soft tissue too is involved by calcium/phosphate deposition. Figure 3 shows this phenomenon where "waves" of calcification can be seen in the muscles of the upper leg.
Patients may develop rapidly progressive heart valve calcification. Ultrafiltration during haemodialysis may be limited by marked falls in blood pressure or patients may develop pulmonary oedema. Many of these patients may not have overtly abnormal serum biochemical profiles.

\section{Inflammation/oxidative stress}

In order to explain the exaggerated prevalence of CVD in patients with CKD, when compared with those without kidney disease, the inflammation/oxidative stress phenomenon has been proposed as a possible additional pathogenetic mechanism. The "confounding by disease" theory ${ }^{(12)}$ allows for debate around the immeasurable process of oxidative stress. The "stress" has been defined as too much damage

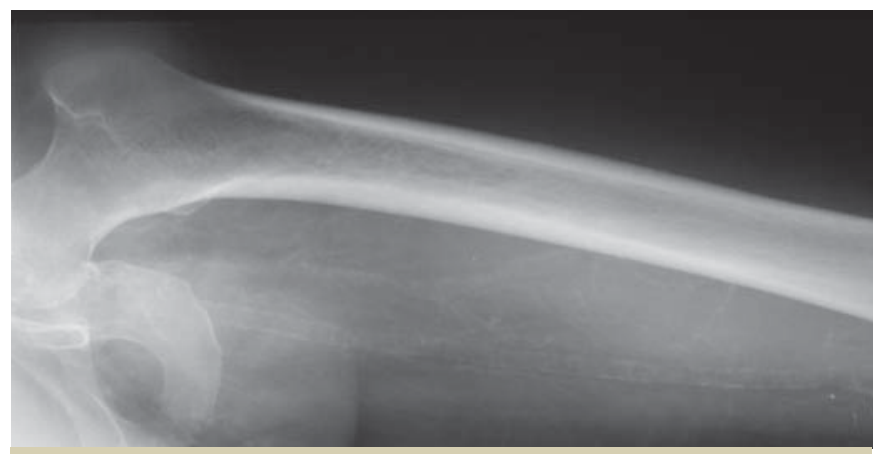

FIGURE 1: an example of both large and small vessel calcification.

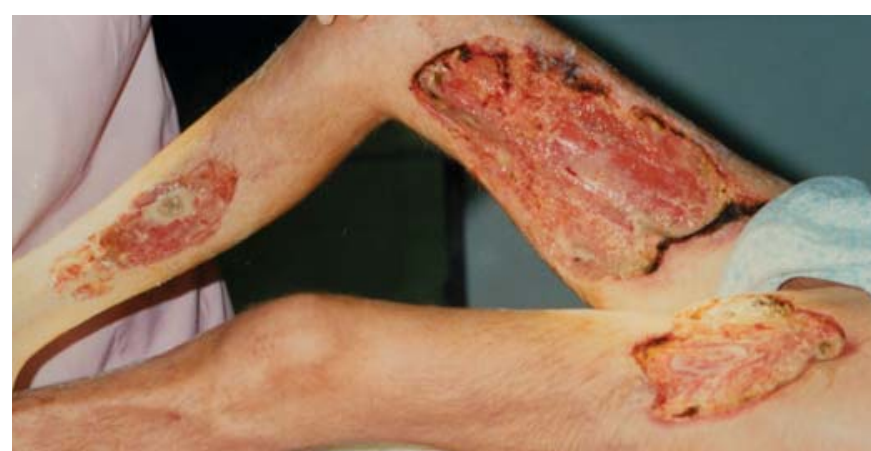

FIGURE 3: A rare complication of small vessel calcification produces systemic calciphylaxis, with skin infarction and progressive ischemic destruction of the subcutaneous tissue.

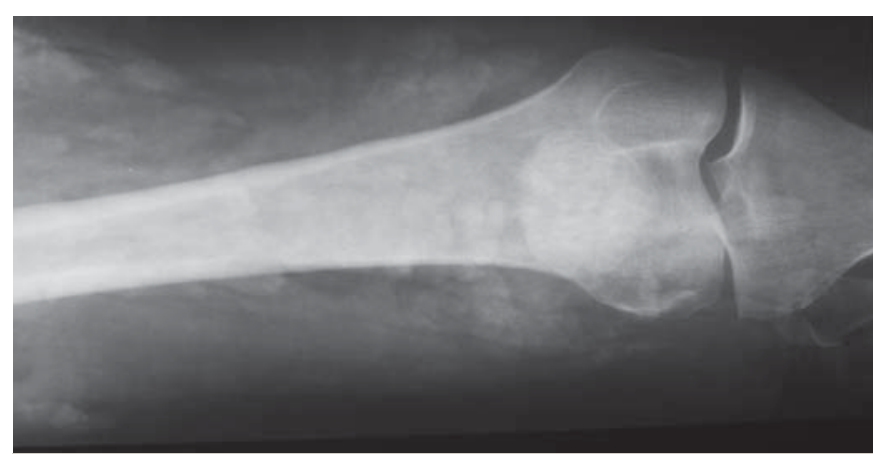

FIGURE 3: Waves of calcification can be seen in the muscles of the upper leg 
to tissues because of too little control. ${ }^{(22)}$ The damage is inflicted by oxidants which are normally used to protect cells from toxins and organisms, but their control, i.e. antioxidant activity, is lacking or inferior. In the uraemic state, the stimulation of the production of oxidants is unchecked and inflammation occurs, with little repair. Under these circumstances atherosclerosis develops. No direct mention is made of arteriosclerosis, but it must be assumed, as for atherosclerosis, that it is similarly influenced by oxidant cell damage.

Macrophages and polymorphonuclear neutrophils produce reactive oxygen species (ROS). ${ }^{(23)} \mathrm{O}_{2}$ is reduced to the superoxide anion, which is immediately changed to hydrogen peroxide $\left(\mathrm{H}_{2} \mathrm{O}_{2}\right)$. The secretion of these oxidants is accompanied by cytokine release, which further impacts on the inflammatory response (and causes injury). The endothelial cell is another source of ROS but is also the major target of these oxidants. ${ }^{(23)}$ Endothelial cell dysfunction permits increasing albuminuria and eventually overt proteinuria, and may allow atherogenic lipids to get into the vessel walls.

The depleted and inefficient anti-oxidant system in CKD comprises, amongst others, superoxide dismutase, vitamin E, vitamin C, ferritin, transferrin and albumin. ${ }^{(23)}$ More still needs to be learnt about the role of these processes in increasing the CV disease risk.

\section{ARRHYTHMIAS}

The risk of arrhythmias in CKD is high. ${ }^{(24)}$ Sudden cardiac death (SCD) is more prevalent in this group because of the presence of hyperkalaemia. This should be the overriding concern of all who manage these patients. Gussak and Gussak ${ }^{(25)}$ propose that the problem is more complex and point to the development of $\mathrm{K}+$ channelopathies in CKD, where coronary artery disease (CAD), left ventricular hypertrophy ( $(\mathrm{VV})$ ) and congestive heart failure (CHF) are associated with a decreased number of ion channels ("diminished repolarisation reserve") but a correspondingly increased sensitivity in the remaining channels. This leads to the development of the acquired long QT syndrome (LQTS) ${ }^{(25)}$ with the risk of torsades de points and SCD. ${ }^{(25)}$

The risk of acquired LQTS is increased by the administration of drugs which prolong the QT interval. This is particularly relevant in the patient with CKD when the drug is wholly, or even partially, excreted by the kidneys.

In haemodialysis patients, the arrhythmias are thought to be related to a brief period of intracellular hypokalaemia and/or hypomagnesaemia whilst on dialysis. ${ }^{(26)}$ Longer dialysis times with a gentler ultrafiltration prescription and a higher $\mathrm{K}^{+}$concentration in the dialysate fluid may be the solution.

\section{THE TREATMENT OF CARDIOVASCULAR DISEASE}

The diagnosis of the acute coronary syndrome (ACS) in CKD is dependent on classical clinical symptoms and signs. ECG evidence is crucial when changes from earlier tracings are evident or obvious ST segment changes are present. It is now well known that cardiac troponin $\mathrm{T}(\mathrm{cTnT})$ is elevated in approximately $25 \%$ of asymptomatic patients with CKD. Its only value, in the setting of acute chest pain, is if the level is within the normal range and myocardial infarction can therefore be excluded. However, Goicoechea et al. have shown that cTnT too is a predictor of $\mathrm{CV}$ events. ${ }^{\left({ }^{6}\right)}$ Their suggestion that further $\mathrm{CV}$ investigations in persons with increased cTnT be undertaken, may be useful. They are supported by an earlier study which concluded that $\mathrm{cTnT}$ elevations were associated with advanced coronary artery disease in asymptomatic patients. ${ }^{(27)}$

De Zeeuw ${ }^{(7,28)}$ and others point out that increasing albuminuria precedes overt renal disease and CVD risk. Angiotensin converting enzyme inhibitors (ACE-Is) and angiotensin receptor blockers (ARBs), by succeeding in reducing albuminuria, also succeed in preserving renal function. They are also cardioprotective. Therefore the use of ACE-Is and $A R B s$ is recommended in patients with increasing albuminuria, overt proteinuria and CKD. More recently, a total blockade of the RAAS has been suggested, whereby an aldosterone antagonist is also prescribed. It was shown that spironolactone may reduce proteinuria and slow progression in patients with CKD. ${ }^{(29)}$ Careful monitoring of $\mathrm{K}^{+}$ levels in patients receiving combinations of these drugs is mandatory.

Control of blood pressure (BP) is very important and is linked to slowing of progression of kidney disease, improved cardiac outcomes and stroke prevention. In the post-hoc analysis of the HOPE study, an ACE-I provided near equal protection against CV events in CKD patients as it did in those with normal renal function. ${ }^{(30)}$

There is ongoing debate on how best to measure BP in CKD. In essential hypertension, ambulatory BP recording better predicts CV mortality and morbidity than clinic readings. ${ }^{(3)}$ In 277 patients with CKD (mean creatinine approximately $180 \mathrm{umol} / \mathrm{I})$, predominantly white men who were elderly and generally obese (average BMI 30.5) and including diabetics (36.9\%), ambulatory BP recordings provided more prognostic information than clinic BP measurements. ${ }^{(32)}$ Systolic BP predicted ESRD compared to total mortality. Night ambulatory BP predicted total mortality. There are only a few such studies in CKD patients, e.g. Liu et al. pointed to non-dipping as being associated with cardiovascular deaths in haemodialysis patients. (33)

Two major publications have recently pointed out the fact that there is very little evidence from controlled studies to give guidance on the treatment of cardiovascular events in patients with CKD. ${ }^{(34,35)}$ 
Approximately $75 \%-80 \%$ of trials examining different coronary artery disease therapies published between 1998 and 2005 excluded patients with CKD. ${ }^{(34)}$ Standard therapy for coronary artery disease may therefore not be effective in patients with CKD. In addition to atherosclerosis, there is also arteriosclerosis and an abnormal metabolic state, all of which may necessitate a different strategy. Patients with CKD need to be included in future intervention studies to answer these difficult management questions.

Edwards et al. provide an excellent review on the treatment of coronary artery disease in CKD. (35) They concede the lack of evidence-based medicine. Referring to patients with CKD, they make the following points:

The beneficial effects of statin therapies are uncertain; however, retrospective examination of data suggests that statin therapy may provide effects similar to those found in patients with normal renal function. So, unless contraindicated, a lipid lowering agent for secondary prevention is indicated. Caution must be exercised in $\mathrm{CKD}$, since rhabdomyolysis is a common complication with the use of stains or fibrates. The SHARP (Study of Heart and Renal Protection) study hopefully will give some finality when the results are published (in 2008).

- The use of beta blockers following ST segment elevation myocardial infarction (STEMI) in CKD patients on haemodialysis is of great advantage and is to be recommended. It must also be remembered that dialysis is a state of great sympathetic over-activity with prominent catecholamine release.

- The revascularisation of coronary arteries by CABG seems to be better than medical treatment or angioplasty. This is not unexpected, since there are metabolic forces in CKD which unrelentingly calcify tissue and stiffen arterial walls.

- Stenting has offered a ray of hope for those patients with an improved long-term prognosis. The PRESTO study is quoted ${ }^{(36)}$ wherein event rates were low and not influenced by GFR. Crude mortality was elevated in the lowest GFR group, but after adjusting for co-morbidity, the difference was insignificant. There has been much discussion on the virtues of drug eluting (anti-proliferative) stents versus non-eluting stents. CKD has not been specifically addressed.

\section{TREATMENT OF THE BONE-MINERAL DISORDER}

An in-depth discussion of this complex issue is beyond the scope of this article. In brief, all attempts must be made to stop the relentless invasion of tissue by calcium and phosphate crystals. The abnormal blood biochemistry, elevated inorganic phosphate and PTH levels, may occur during the early stages of CKD. The treatment at these earlier stages is uncertain. There are cost implications, as well as exposure to excessive calcium (through calcium-containing phosphate binders and the use of vitamin D) to consider in designing the treatment strategy. Taking calcium carbonate and vitamin D for many years risks excessive exposure to calcium and the possibility of eventual vascular calcification. The treatment of these biochemical abnormalities must be individualised, preferably with input from a nephrologist.

\section{TREATMENT OF OBESITY}

The fact that there appears to be an improved survival of obese patients on haemodialysis does not mean that it should be encouraged in the lead-up to dialysis. What it does suggest is that malnutrition and very low BMls should be avoided, hence caution is indicated in advising protein restriction in an attempt to delay the progression of CKD.

Progression to stage 5 CKD (ESRD) is accelerated in obesity; it is associated with insulin resistance, diabetes and hypertension, and is a defining factor for the metabolic syndrome. The metabolic syndrome is a risk factor for CVD. ${ }^{(37)}$ Every effort should therefore be made to avoid obesity but not permit the development of excessive loss of weight or malnourishment.

\section{CONCLUSION}

CKD is clearly a major risk factor for CVD. The traditional risk factors should be managed according to current guidelines. ACE-Is and ARBs must remain the mainstay of treatment of hypertension, and should also be used to reduce proteinuria and to slow the progression to endstage kidney disease.

In the patient with CKD, bone-mineral disorder is particularly relevant in contributing to vascular disease, and its management requires longterm surveillance by an experienced physician or nephrologist.

Randomised controlled studies are awaited to assess the best approach to CKD patients with coronary artery disease. As these patients have been excluded from most major intervention studies, the only information available is derived from registries or smaller controlled studies. 


\section{REFERENCES:}

I. K/DOQI clinical practice guidelines for chronic kidney disease: evaluation, classification and stratification. Kidney Diseases Outcome Quality Initiative. Am J Kidney Dis Suppl I 2002;39:SI-S246

2. Couser G. A call to action on World Kidney Day, 8 March 2007. Kidney Int 2007;71: 369-370.

3. Baigent C, Burbury K, Wheeler D. Premature cardiovascular disease in chronic renal failure. Lancet 2000;356:147-142.

4. Culleton BF, Larson NGM, Wilson PWF et al. Cardiovascular disease and mortality in a community-based cohort with mild renal insufficiency. Kidney Int 1999;56:2214-2219.

5. Go AS, Cherton GM, Fan D et al. Chronic kidney disease and the risks of death, cardiovascular events and hospitalisation. N Eng J Med 2004;35 |: | 296- | 305.

6. Goicoechea M, DeVinuesa SG, Gomez-Campdera F et al. Predictive cardiovascular risk factors in patients with chronic kidney disease (CKD). Kidney Int 2005;67-5933: 535-538

7. De Zeeuw D. Albuminuria, not only a cardiovascular renal risk marker, but also a target for treatment? Kidney Int 2004;66,(Supp 92):S2-S6

8. Wang Z, Hoy WE. Albuminuria and incident coronary heart disease in Australian Aboriginal people. Kidney Int 2005;68: 1289-1293.

9. Dickert T, Feldt-Rasmussen B, Borch-Johnsen $\mathrm{K}$ et al. Albuminuria reflects widespread vascular damage. The Steno hypothesis. Diabetologia 1989;32:219-226.

10. Beddhu S.The body mass index paradox and an obesity, inflammation and atheroscerlosis syndrome in chronic kidney disease. Semin Dial 2004;17(3):229-232.

I I. Kalantar-Zadeli K and Kopple JD. Obesity paradox in patients on maintenance dialysis. Contrib Nephrol 2006; 151:57-69.

12 Baigent $C$ and Landray $M$. Which cardiovascular risk factors matter in chronic kidney disease? Nephrol Dial Transplant 2007;22:9-1।.

13 Sarnak MJ and Levey AS. Cardiovascular disease and chronic renal disease: a new paradigm. Am J Kidney Dis 2000;35:SI I7-SI 31.

I4 Longenecker JC, Klag MJ, Marcovina SM et al. High lipoprotein (a) levels and small apolipoprotein (a) size prospectively predict cardiovascular events in dialysis patients. J Am Soc Nephrol 2005; 16:1794 -

15. Lewington S, Clarke R, Qizilbash $N$ et al. Age-specific relevance of usual blood pressure to vascular mortality: a meta analysis of individual data for one million adults in 6 prospective studies. Lancet 2002;360:1903-1913.

16. Rirz E. Hypertension: The kidney is the culprit even in the absence of kidney disease. Kidney Int 2007;71:371-372

17. Bremner BM, Garcia DL, Anderson S. Glomeruli and blood pressure. Less of one, more of the other? Am J Hypertens 1988; 1:335-347.

18. Verberckmoes SC, Persy V, Behets G] et al. Uremia-related vascular calcification: More than apatite deposition. Kidney Int 2007;71:298-303

19. O'Neill WC.Vascular calcification: Not so crystal clear. Kiney Int 2007;71:282-283.

20 Reynolds JL, Joannides AJ, Skepper JN et al. Human vascular smooth muscle cells undergo vesicle-mediated calcification in response to changes in extracellular calcium and phosphate concentrations: a potential mechanism for accelerated vascular calcification in ESRD. J Am Soc Nephrol 2004;15:2857-2867.

21. Goodman WG, Goldin J, Kuizon BD et al. Coronary-artery calcification in young adults with end-stage renal disease who are undergoing dialysis. N Engl | Med 2000;342: |478-| 483.

22. Sies H. Oxidants and antioxidants. Exp Physiol 1997;82:291-295.

23. Locatelli F, Canaud B, Eckardt $K$ et al. Oxidative stress in end-stage renal disease: an emerging threat to patient outcome. Nephrol Dial Transplant 2003; 1 8: 1272-1280.

24. Tonelli M, Wiebe N, Culleton B et al. Chronic kidney disease and mortality risk: a systematic review. J Am Soc Nephrol 2006; 17:2034-2047.

25. Gussak I and Gussak HM. Sudden cardiac death in nephrology: focus on acquired long QT syndrome. Nephrol Dial Transplant 2007;22:12-14.

26. Curtis $\mathrm{LH}$, Ostbye T, Sendersky $\vee$ et al. Prescription of QT-prolonging drugs in a cohort of about 5 million outpatients. Am J Med 2003; | | 4: | 35- | 41.

27. De Filipi C,Wasserman S, Rosanio S et al. Cardiac troponin R and C-reactive protein for predicting prognosis, coronary atherosclerosis and cardiomyopathy in patients undergoing long-term haemodialysis. JAMA 2003; 290: 35x-359.

28. Hillege $\mathrm{HL}$, Janssen WM, BakAA et al. Microalbuminuria is common, also in a nondiabetic, nonhypertensive population and an independent indicator of cardiovascular risk factors and cardiovascular morbidity. J Int Med 2001;249:5 19-526.
29. Bianchi $S$ and Campese VM. Long-term effects of spironolactone on proteinuria and kidney function in patients with chronic kidney disease. Kidney Int 2006;70:21 16-2123.

30. Mann JF, Gerstein HC, Pogue J et al. Renal insufficiency as a predictor of cardiovascular outcomes and the impact of ramipril: the HOPE randomized trial. Ann Intern Med 2001; 134:629-636.

31. Clement DL, De Buyzere ML, De Bacquer DA et al. Prognostic value of ambulatory blood-pressure recordings in patients with treated hypertension. N Engl J Med 2003:348:2407-2415.

32. Agarwal R and Andersen MJ. Prognostic importance of ambulatory blood pressure recordings in patients with chronic kidney disease. Kidney Int 2006;69:1 I 75- I I 80.

33. Liu M, Takahashi H. Morita Y. Non-dipping is a potent predictor of cardiovascular mortality and is associated with autonomic dysfunction in haemodialysis patients. Nephrol Dial Transplant 2003; 1 8:563-569.

34. Charytan D and Kuntz RE. The exclusion of patients with chronic kidney disease from clinical trials in coronary artery disease. Kidney Int 2006;70:202 I-2030.

35. Edwards NC, Steeds RP, Ferro C], Townend JN. The treatment of coronary artery disease in patients with chronic kidney disease. Q J Med 2006:99:723-736.

36. Best PJ, Berger PB, Davis BR et al. Impact of mild or moderate chronic kidney disease on the frequency of restenosis: results from the PRESTO trial. J Am Coll Cardiol 2004; 44:1786-179|.

37. Mark DH. Deaths attributable to obesity. JAMA 2005;293:1918-1919. 